\title{
A new proof of completeness for a relative modal logic with composition and intersection
}

\author{
Philippe Balbiani \\ Laboratoire d'informatique de Paris-Nord*
}

\begin{abstract}
This paper is devoted to the completeness issue of $R M L C I-$ the relative modal logic with composition and intersection - a restriction of the propositional dynamic logic with intersection. The trouble with $R M L C I$ is that the operation of intersection is not modally definable. Using the notion of mosaics, we give a new proof of a theorem considered in a previous paper "Complete axiomatization of a relative modal logic with composition and intersection". The theorem asserts that the proof theory of $R M L C I$ is complete for the standard Kripke semantics of $R M L C I$.
\end{abstract}

\section{Introduction}

A relative modal logic is a modal logic the modal operators of which depend on parameters. Among the well-known relative modal logics devised in artificial intelligence and computer science, there are $P D L$ - the propositional dynamic logic - introduced by Fischer and Ladner [6], $P A L$ - the propositional algorithmic logic — set out by Mirkowska [12], $D A L$ — the logic for data analysis - expounded by Fariñas del Cerro and Orłowska [5], and $B M L$ - the Boolean modal logic — brought in by Gargov and Passy [7].

*Philippe Balbiani, balbiani@lipn.univ-paris13.fr, Laboratoire d'informatique de ParisNord, Institut Galilée, Université Paris-Nord, 99 avenue Jean-Baptiste Clément, F-93430 Villetaneuse. 
Recently, artificial intelligence and computer science have also given rise to several relative modal logics for reasoning about common knowledge in a distributed environment [4]. Beyond doubt, $P D L$ is par excellence an applied non-classical logic with which it is possible to reason about programs. Its linguistic basis is the propositional calculus enlarged with a family of modal operators of the form $[a]$ where $a$ is a program composed by induction via regular rules in the following way : $a=\pi \mid A$ ? $|a ; b| a \vee b \mid a^{\star}$ where $\pi$ ranges over the set of the atomic programs and $A$ ranges over the set of the formulas. Semantically, a program $a$ corresponds to a binary relation of accessibility $R(a)$ between the states of some presumed universe $W$, seeing that $R(A$ ? $)=$ $\{(x, x)$ : the formula $A$ is true in the state $x\}, R(a ; b)=R(a) \circ R(b), R(a \vee b)$ $=R(a) \cup R(b)$ and $R\left(a^{\star}\right)=R(a)^{\star}$.

Amid the multifarious restrictions and extensions of $P D L$, this paper will take an interest in $R M L C I$ - the relative modal logic with composition and intersection - which programs are composed by induction via regular rules in the following way : $a=\pi|a ; b| a \wedge b$, seeing that $R(a \wedge b)=R(a) \cap R(b)$. $R M L C I$ is a relative modal logic which decidability is an outcome of the decidability of $I P D L$ - the propositional dynamic logic with intersection - demonstrated by Danecki [3]. The complete axiomatisation of RMLCI is feasible by means of special atomic formulas called names to be interpreted as true at exactly one state [14]. The question of a name-free axiomatisation of the concept of validity in all models of $R M L C I$ is interesting because composition and intersection figure prominently in several relative modal logics for which the issue of their complete axiomatization remains to be solved. To illustrate the truth of this, one may remark that $R M L C I$ is a special case of a more general notion of a relative modal logic which has been expounded by Orłowska [13] in the context of incomplete information systems. As well, one may observe that $R M L C I$ is a fragment of a deontic logic set out by Meyer [11]. The fact of the matter is that the question of a name-free axiomatisation of the concept of validity in all models of $R M L C I$ is quite an undertaking in view of the inability of modal logic to define the condition $R(a \wedge b) \supseteq R(a) \cap R(b)$. In all probability, the obstacles encountered by those who first tried to axiomatise the concept of validity in all models of $I P D L$ have something to do with this incapacity.

In a previous paper "Complete axiomatization of a relative modal logic with composition and intersection", we have pursued the question of whether the concept of validity in all models of $R M L C I$ can be elegantly axiomatised. 
Backing up our line of reasoning with the techniques of the subordination introduced by Cresswell [9], then we have succeeded in proving the completeness of some axiomatisation of our relative modal logic with composition and intersection. Seeing that we have a few reservations about the portability of our proof of completeness, our object in writing this paper is to lay out a new proof of completeness for $R M L C I$ which is based on the techniques of the mosaics brought in by Nemeti and furthered by Marx [10]. The syntax of $R M L C I$ is presented in the section 2 whereas the semantics of $R M L C I$ is presented in the section 3. The axiomatization of $R M L C I$ is expounded in the section 4 whereas the proof of the completeness of the axiomatization of $R M L C I$ is organized in the sections 5 and 6 .

\section{Syntax}

What we are mainly concerned with here is the syntax of $R M L C I$. Let us consider a countable set $\Pi_{a}$ of atomic programs as well as a countable set $\Phi_{a}$ of atomic formulas. The set $\Pi_{c}$ of the programs as the set $\Phi_{c}$ of the formulas are generated by the following recursive equations where $\pi$ ranges over $\Pi_{a}$ and $P$ ranges over $\Phi_{a}: a=\pi|a ; b| a \wedge b$ and $A=P|\perp| A \rightarrow B \mid[a] A$. Other connectives are introduced by the usual abbreviations.

\section{Semantics}

Let us now look at the semantics of $R M L C I$. In order to clear the ground, let us give ourselves the means to compare programs between themselves. In the first place, let $\equiv$ be the least congruence on $\Pi_{c}$ such that $a ;(b ; c) \equiv$ $(a ; b) ; c, a \wedge(b \wedge c) \equiv(a \wedge b) \wedge c, a \wedge b \equiv b \wedge a$ and $a \wedge a \equiv a$. In the second place, let $\leq$ be the binary relation on $\Pi_{c}$ such that $a \leq b$ iff $a \wedge b \equiv a$. We commonly think of frames as relational structures of the form $(W, R)$ where $W$ is a non-empty set of states and $R$ is a relation of accessibility that maps $\Pi_{c}$ to the set of the binary relations on $W$. More exactly, it is necessary that $R$ satisfies the following conditions for $(W, R)$ to be called strong frame: $R(a ; b)=R(a) \circ R(b)$ and $R(a \wedge b)=R(a) \cap R(b)$ whereas it is necessary that $R$ satisfies the following conditions for $(W, R)$ to be called weak frame: $R(a ; b)=R(a) \circ R(b)$ and if $a \leq b$ then $R(a) \subseteq R(b)$. The following lemma 
holds :

Lemma 1 Let $a, b$ be programs. $a \equiv b$ iff, for all strong frame $(W, R), R(a)$ $=R(b)$.

Proof : Cf. Bredikhin and Schein [2]. $\dashv$

It follows immediately that :

Lemma 2 Every strong frame is a weak frame.

Proof : We leave the proof to the reader. $\dashv$

What is more :

Lemma 3 The problem of proving, for all programs $a, b$, whether $a \equiv b$ or not is decidable.

Proof : By showing that expressions of the form $a \equiv b$ correspond to formulas in the decidable $\exists^{\star} \forall^{\star}$ fragment of first order logic, the Bernays-Schönfinkel class. $\dashv$

A model is a relational structure of the form $(W, R, V)$ where $(W, R)$ is a frame and $V$ is a function that maps $\Phi_{c}$ to the set of the subsets of $W$ such that : $V(\perp)=\emptyset, V(A \rightarrow B)=\{x:$ if $x \in V(A)$ then $x \in V(B)\}$ and $V([a] A)$ $=\{x:$ for all $y \in W$, if $x R(a) y$ then $y \in V(A)\}$. A formula $A$ is said to be valid in $(W, R, V)$ if it satisfies the following condition : $V(A)=W$. Strong models constitute the standard Kripke semantics of RMLCI whereas weak models make up the non-standard Kripke semantics of RMLCI. This brings us to the question of whether we can axiomatise the concept of validity in the standard Kripke semantics of $R M L C I$. It would hardly be an exaggeration to say that in reality the issue is an extremely complex one. For the simple reason that the condition $R(a \wedge b) \supseteq R(a) \cap R(b)$ is not modally definable. On the other hand, thanks to the modal definability of the conditions $R(a ; b)=$ $R(a) \circ R(b)$ and $R(a) \subseteq R(b)$, there is no doubt that we can easily axiomatise the concept of validity in the non-standard Kripke semantics of $R M L C I$. In this respect, what we have in mind is to use the concept of p-morphism and demonstrate that every weak frame is a p-morphic image of a strong frame. For all frames $(W, R),\left(W^{\prime}, R^{\prime}\right)$ and for all function $f$ that maps $W$ to $W^{\prime}$, if $f$ satisfies the following condition then $f$ is called homomorphism of $(W, R)$ to $\left(W^{\prime}, R^{\prime}\right)$ while $\left(W^{\prime}, R^{\prime}\right)$ is called homomorphic image of $(W, R)$ : for all $x, y$ $\in W$, if $x R(a) y$ then $f(x) R^{\prime}(a) f(y)$. If, moreover, $f$ satisfies the following 
conditions then $f$ is called p-morphism of $(W, R)$ to $\left(W^{\prime}, R^{\prime}\right)$ while $\left(W^{\prime}, R^{\prime}\right)$ is called p-morphic image of $(W, R)$ : for all $x^{\prime} \in W^{\prime}$, there exists $x \in W$ such that $f(x)=x^{\prime}$ and, for all $x \in W$ and for all $y^{\prime} \in W^{\prime}$, if $f(x) R^{\prime}(a) y^{\prime}$ then there exists $y \in W$ such that $x R(a) y$ and $f(y)=y^{\prime}$. The following result can be obtained :

Lemma 4 Let $(W, R),\left(W^{\prime}, R^{\prime}\right)$ be frames, $f$ be a p-morphism of $(W, R)$ to $\left(W^{\prime}, R^{\prime}\right), V$ be a valuation on $(W, R)$ and $V^{\prime}$ be a valuation on $\left(W^{\prime}, R^{\prime}\right)$ such that, for all atomic formula $P, V(P)=\left\{x: f(x) \in V^{\prime}(P)\right\}$. For all formula $A, V(A)=\left\{x: f(x) \in V^{\prime}(A)\right\}$.

Proof : This is the p-morphism lemma, a standard result. $\dashv$

\section{Proof theory}

Now is the time to expound the proof theory of $R M L C I$. The issue at stake here is one of syntactic characterization of the concept of validity in the standard Kripke semantics of $R M L C I$. Together with the classical axioms, all the instances of the following schemata are axioms of RMLCI : $[a](A \rightarrow$ $B) \rightarrow([a] A \rightarrow[a] B),\langle a ; b\rangle A \leftrightarrow\langle a\rangle\langle b\rangle A$ and if $a \leq b$ then $\langle a\rangle A \rightarrow\langle b\rangle A$. Together with the classical rules of inference, all the instances of the following schema are rules of inference of $R M L C I$ : from $A$ infer $[a] A$. It is well worth noting that the use of the binary relation $\leq$ in the proof theory of $R M L C I$ has a lot to do with the use of the binary relation $\subseteq$ in the proof theory of $B M L$ brought in by Gargov and Passy [7]. The following theorem holds :

Theorem 1 If a formula is valid in all weak models then it is a theorem of $R M L C I$.

Proof : By showing that the canonical model of $R M L C I$ is a weak model. $\dashv$ What is more :

Proposition 1 The set of the axioms of RMLCI is recursive.

Proof : By lemma 3. $\dashv$

Finally, there is the related problem of how to eliminate the use of the binary relation $\leq$ in the proof theory of $R M L C I$. Together with the classical axioms, all the instances of the following schemata are axioms of $R M L C I^{\prime}$ : 
$[a](A \rightarrow B) \rightarrow([a] A \rightarrow[a] B),\langle a ; b\rangle A \leftrightarrow\langle a\rangle\langle b\rangle A,\langle a \wedge b\rangle A \rightarrow\langle a\rangle A$ and $\langle a \wedge b\rangle A \rightarrow\langle b\rangle A$. Together with the classical rules of inference, all the instances of the following schemata are rules of inference of $R M L C I^{\prime}$ : from $A$ infer $[a] A$ and from $[a] P \vee[b] P \rightarrow[c] P$ infer $[a \wedge b] A \rightarrow[c] A$. The following theorems hold :

Theorem 2 If a formula is a theorem of RMLCI then it is a theorem of $R M L C I^{\prime}$.

Proof : The easy proof that, for all programs $a, b, a \equiv b$, and for all formula $A,\langle a\rangle A \rightarrow\langle b\rangle A$ is a theorem of $R M L C I^{\prime}$ and $\langle b\rangle A \rightarrow\langle a\rangle A$ is a theorem of $R M L C I^{\prime}$ is left to the reader. Now let $a, b$ be programs, $a \leq b$, and $A$ be a formula. Since $a \leq b$, then $a \wedge b \equiv a$ and $\langle a\rangle A \rightarrow\langle a \wedge b\rangle A$ is a theorem of $R M L C I^{\prime}$. Since $\langle a \wedge b\rangle A \rightarrow\langle b\rangle A$ is an axiom of $R M L C I^{\prime}$, then $\langle a\rangle A \rightarrow\langle b\rangle A$ is a theorem of $R M L C I^{\prime} . \dashv$

Theorem 3 If a formula is a theorem of $R M L C I^{\prime}$ then it is valid in all strong models.

Proof : By demonstrating that every axiom of $R M L C I^{\prime}$ is valid in all strong models and that the rules of inference of $R M L C I^{\prime}$ preserve validity for the class of all strong models. $\dashv$

However, we do not know whether or not a formula valid in all strong models is also valid in all weak models. The truth of the matter is that there is no easy answer to the issue at stake here. So we will not consider this question before the last section of this paper.

\section{$5 \quad$ Mosaics}

With a view to proving that a formula valid in all strong models is also valid in all weak models, we have decided to demonstrate that every weak frame is a p-morphic image of a strong frame. This plan necessitates the use of the concept of mosaic. Let $\downarrow$ be the partial function that maps $\Pi_{c} \times \mathbb{R} \times \mathbb{R}$ to the set of the frames which is defined by induction on the formation rules for programs in the following way. First of all, for all atomic program $\pi$ and for all real numbers $x, y, x \neq y$, let $\downarrow(\pi, x, y)$ be the frame of the form $(W, R)$ where $W=\{x, y\}$ and, for all program $c$ and for all $t, u \in W, t R(c) u$ iff : 
- $t=x, u=y$ and $\pi \leq c$.

Secondly, for all programs $a, b$ and for all real numbers $x, y, x \neq y$, let $\natural(a ; b, x, y)$ be the frame of the form $(W, R)$ where $z$ is a new real number, $\downarrow(a, x, z)=\left(W_{a}, R_{a}\right), \downarrow(b, z, y)=\left(W_{b}, R_{b}\right), W=W_{a} \cup W_{b}$ and, for all program $c$ and for all $t, u \in W, t R(c) u$ iff :

- either $t, u \in W_{a}$ and $t R_{a}(c) u$

- or $t \in W_{a} \backslash\{z\}, u \in W_{b} \backslash\{z\}$ and there exist programs $c^{\prime}, c^{\prime \prime}$ such that $t R_{a}\left(c^{\prime}\right) z, z R_{b}\left(c^{\prime \prime}\right) u$ and $c^{\prime} ; c^{\prime \prime} \leq c$

- or $t, u \in W_{b}$ and $t R_{b}(c) u$.

Third, for all programs $a, b$ and for all real numbers $x, y, x \neq y$, let $\natural(a \wedge b, x, y)$ be the frame of the form $(W, R)$ where $\downarrow(a, x, y)=\left(W_{a}, R_{a}\right), \downarrow(b, x, y)=$ $\left(W_{b}, R_{b}\right), W=W_{a} \cup W_{b}$ and, for all program $c$ and for all $t, u \in W, t R(c)$ $u$ iff :

- either $t, u \in W_{a}, t \neq x$ or $u \neq y$, and $t R_{a}(c) u$

- or $t=x, u=y$ and $a \wedge b \leq c$

- or $t, u \in W_{b}, t \neq x$ or $u \neq y$, and $t R_{b}(c) u$.

For all program $a$ and for all real numbers $x, y, x \neq y, \downarrow(a, x, y)$ is called mosaic of a with respect to $x$ and $y$. Let us remember that:

Lemma 5 Let $\left(W^{\prime}, R^{\prime}\right)$ be a weak frame, a be a program, $x, y$ be real numbers, $x \neq y$, and $x^{\prime}, y^{\prime} \in W^{\prime}, x^{\prime} R^{\prime}(a) y^{\prime}$. There exists a homomorphism $h$ of $\natural(a, x, y)$ to $\left(W^{\prime}, R^{\prime}\right)$ such that $h(x)=x^{\prime}$ and $h(y)=y^{\prime}$.

Proof : By induction on $a . \dashv$

Our aim is to demonstrate that mosaics are finite irreflexive strong frames. In this respect, we need to consider the following lemmas :

Lemma 6 Let a be a program and $x, y$ be real numbers, $x \neq y$. $\downarrow(a, x, y)$ is a finite irreflexive frame of the form $(W, R)$ such that $x, y \in W$, for all program $c, x R(c)$ y iff $a \leq c$ and $R$ satisfies the conditions : $R(c ; d) \supseteq R(c) \circ R(d)$ and $R(c \wedge d)=R(c) \cap R(d)$. 
Proof : By induction on $a . \dashv$

Lemma 7 Let $a, b$ be programs, $a \geq b$, and $x, y, x^{\prime}, y^{\prime}$ be real numbers, $x \neq$ $y$ and $x^{\prime} \neq y^{\prime}$. There exists a homomorphism $h$ of $\downarrow(a, x, y)$ to $\downarrow\left(b, x^{\prime}, y^{\prime}\right)$ such that $h(x)=x^{\prime}$ and $h(y)=y^{\prime}$.

Proof : The easy proof that, for all programs $a, b, a \equiv b$, and for all real numbers $x, y, x^{\prime}, y^{\prime}, x \neq y$ and $x^{\prime} \neq y^{\prime}$, there exists a homomorphism $h$ of $\downarrow(a, x, y)$ to $\downarrow\left(b, x^{\prime}, y^{\prime}\right)$ such that $h(x)=x^{\prime}$ and $h(y)=y^{\prime}$ and there exists a homomorphism $h^{\prime}$ of $\downarrow\left(b, x^{\prime}, y^{\prime}\right)$ to $\downarrow(a, x, y)$ such that $h^{\prime}\left(x^{\prime}\right)=x$ and $h^{\prime}\left(y^{\prime}\right)=$ $y$ is left to the reader. Now let $a, b$ be programs, $a \geq b$, and $x, y, x^{\prime}, y^{\prime}$ be real numbers, $x \neq y$ and $x^{\prime} \neq y^{\prime}$. Since $a \geq b$, then $a \wedge b \equiv b$ and there exists a homomorphism $h$ of $\downarrow(a \wedge b, x, y)$ to $\natural\left(b, x^{\prime}, y^{\prime}\right)$ such that $h(x)=x^{\prime}$ and $h(y)$ $=y^{\prime}$. Since $\sharp(a \wedge b, x, y)$ is the frame of the form $(W, R)$ where $\downarrow(a, x, y)=$ $\left(W_{a}, R_{a}\right),\left\llcorner(b, x, y)=\left(W_{b}, R_{b}\right), W=W_{a} \cup W_{b}\right.$ and, for all program $c$ and for all $t, u \in W, t R(c) u$ iff :

- either $t, u \in W_{a}, t \neq x$ or $u \neq y$, and $t R_{a}(c) u$

- or $t=x, u=y$ and $a \wedge b \leq c$

- or $t, u \in W_{b}, t \neq x$ or $u \neq y$, and $t R_{b}(c) u$,

then the reader may easily verify that $h_{\mid W_{a}}$ is a homomorphism of $\downarrow(a, x, y)$ to $\natural\left(b, x^{\prime}, y^{\prime}\right)$ such that $h_{\mid W_{a}}(x)=x^{\prime}$ and $h_{\mid W_{a}}(y)=y^{\prime}$.

Lemma 8 Let a be a program, $x^{\prime}, y^{\prime}$ be real numbers, $x^{\prime} \neq y^{\prime}, \downarrow\left(a, x^{\prime}, y^{\prime}\right)$ be a frame of the form $\left(W^{\prime}, R^{\prime}\right)$, c be a program, $t, u$ be real numbers, $t \neq u$, and $t^{\prime}, u^{\prime} \in W^{\prime}, t^{\prime} R^{\prime}(c) u^{\prime}$. There exists a homomorphism $h$ of $\downarrow(c, t, u)$ to $\left(W^{\prime}, R^{\prime}\right)$ such that $h(t)=t^{\prime}$ and $h(u)=u^{\prime}$.

Proof : By induction on $a$. We should just like to point out that the proof of lemma 8 makes use of lemma 7 . $\dashv$

Lemma 9 Let a be a program and $x^{\prime}, y^{\prime}$ be real numbers, $x^{\prime} \neq y^{\prime}$. $\llbracket\left(a, x^{\prime}, y^{\prime}\right)$ is a frame of the form $\left(W^{\prime}, R^{\prime}\right)$ such that $R^{\prime}$ satisfies the condition : $R^{\prime}(c ; d)$ $\subseteq R^{\prime}(c) \circ R^{\prime}(d)$.

Proof : Let $c, d$ be programs and $t^{\prime}, u^{\prime} \in W^{\prime}, t^{\prime} R^{\prime}(c ; d) u^{\prime}$. Suppose now that, for all $z^{\prime} \in W^{\prime}, t^{\prime} \overline{R^{\prime}(c)} z^{\prime}$ or $z^{\prime} \overline{R^{\prime}(d)} u^{\prime}$. Let $t, u$ be real numbers, $t$ 
$\neq u$. Let $\downarrow(c ; d, t, u)$ be the frame of the form $(W, R)$ where $z$ is a new real number, $\downarrow(c, t, z)=\left(W_{c}, R_{c}\right), \downarrow(d, z, u)=\left(W_{d}, R_{d}\right), W=W_{c} \cup W_{d}$ and, for all program $e$ and for all $p, q \in W, p R(e) q$ iff :

- either $p, q \in W_{c}$ and $p R_{c}(e) q$

- or $p \in W_{c} \backslash\{z\}, q \in W_{d} \backslash\{z\}$ and there exist programs $e^{\prime}, e^{\prime \prime}$ such that $p R_{c}\left(e^{\prime}\right) z, z R_{d}\left(e^{\prime \prime}\right) q$ and $e^{\prime} ; e^{\prime \prime} \leq e$

- or $p, q \in W_{d}$ and $p R_{d}(e) q$.

By lemma 8, there exists a homomorphism $h$ of $(W, R)$ to $\left(W^{\prime}, R^{\prime}\right)$ such that $h(t)=t^{\prime}$ and $h(u)=u^{\prime}$. Since $t R_{c}(c) z$ then $t R(c) z$. Since $z R_{d}(d) u$ then $z R(d) u$. Then $t^{\prime} R^{\prime}(c) h(z)$ and $h(z) R^{\prime}(d) u^{\prime}$, a contradiction. $\dashv$

It follows immediately that :

Proposition 2 Let a be a program and $x, y$ be real numbers, $x \neq y$. $\downarrow(a, x, y)$ is a finite irreflexive strong frame of the form $(W, R)$ such that $x, y \in W$ and, for all program $c, x R(c)$ y iff $a \leq c$.

Proof : By lemmas 6 and 9. $\dashv$

\section{Completeness}

Our intention is to prove that a formula valid in all strong models is also valid in all weak models. Accordingly, we have no alternative but to demonstrate that :

Lemma 10 Every weak frame is a p-morphic image of an irreflexive strong frame.

Proof : Let $\left(W^{\prime}, R^{\prime}\right)$ be a weak frame. We use mosaics as components to build up step by step an irreflexive strong frame $(W, R)$ and a p-morphism $f$ of $(W, R)$ to $\left(W^{\prime}, R^{\prime}\right)$. Let $\left(W_{0}, R_{0}\right),\left(W_{1}, R_{1}\right), \ldots$ be a sequence of frames and $f_{0}, f_{1}, \ldots$ be a sequence of functions that maps $W_{0}, W_{1}, \ldots$ to $W^{\prime}$ defined by induction in the following way.

Basis : Let :

- $W_{0}=W^{\prime}$, 
- $R_{0}$ be the function that maps $\Pi_{c}$ to the set of the binary relations on $W_{0}$ such that, for all program $a$ and for all $x, y \in W_{0}, x \overline{R_{0}(a)} y$ and

- $f_{0}$ be the function that maps $W_{0}$ to $W^{\prime}$ such that, for all $x \in W_{0}, f_{0}(x)$ $=x$.

It should be remarked that $\left(W_{0}, R_{0}\right)$ is an irreflexive strong frame and $f_{0}$ is a surjective homomorphism of $\left(W_{0}, R_{0}\right)$ to $\left(W^{\prime}, R^{\prime}\right)$.

Hypothesis : Let $i$ be a positive integer, $\left(W_{i}, R_{i}\right)$ be an irreflexive strong frame and $f_{i}$ be a surjective homomorphism of $\left(W_{i}, R_{i}\right)$ to $\left(W^{\prime}, R^{\prime}\right)$. What is more, let $c$ be a program, $z \in W_{i}$ and $t^{\prime} \in W^{\prime}$ be such that $f_{i}(z) R^{\prime}(c)$ $t^{\prime}$. In that case, $\left(c, z, t^{\prime}\right)$ is called defect of $\left(W_{i}, R_{i}\right)$ with respect to $\left(W^{\prime}, R^{\prime}\right)$ and $f_{i}$. For all defect $\left(c, z, t^{\prime}\right)$ of $\left(W_{i}, R_{i}\right)$ with respect to $\left(W^{\prime}, R^{\prime}\right)$ and $f_{i}$, let $\natural(c, z, t)$ be the frame of the form $\left(W_{c}^{z, t}, R_{c}^{z, t}\right)$ where $t$ is a new real number. By lemma 5, there exists a homomorphism $h_{c}^{z, t}$ of $\left(W_{c}^{z, t}, R_{c}^{z, t}\right)$ to $\left(W^{\prime}, R^{\prime}\right)$ such that $h_{c}^{z, t}(z)=f_{i}(z)$ and $h_{c}^{z, t}(t)=t^{\prime}$.

Step : Our aim is to put right the defects of $\left(W_{i}, R_{i}\right)$ with respect to $\left(W^{\prime}, R^{\prime}\right)$ and $f_{i}$. In this respect, let :

- $W_{i+1}=W_{i} \cup \bigcup\left\{W_{c}^{z, t}:\left(c, z, t^{\prime}\right)\right.$ is a defect of $\left(W_{i}, R_{i}\right)$ with respect to $\left(W^{\prime}, R^{\prime}\right)$ and $\left.f_{i}\right\}$

- $R_{i+1}$ be the function that maps $\Pi_{c}$ to the set of the binary relations on $W_{i+1}$ such that, for all program $a$ and for all $x, y \in W_{i+1}, x R_{i+1}(a) y$ iff :

- either $x, y \in W_{i}$ and $x R_{i}(a) y$

- or there exists a defect $\left(c, z, t^{\prime}\right)$ of $\left(W_{i}, R_{i}\right)$ with respect to $\left(W^{\prime}, R^{\prime}\right)$ and $f_{i}$ such that $x \in W_{i} \backslash\{z\}, y \in W_{c}^{z, t} \backslash\{z\}$ and there exist programs $a^{\prime}, a^{\prime \prime}$ such that $x R_{i}\left(a^{\prime}\right) z, z R_{c}^{z, t}\left(a^{\prime \prime}\right) y$ and $a^{\prime} ; a^{\prime \prime} \leq a$

- or there exists a defect $\left(c, z, t^{\prime}\right)$ of $\left(W_{i}, R_{i}\right)$ with respect to $\left(W^{\prime}, R^{\prime}\right)$ and $f_{i}$ such that $x, y \in W_{c}^{z, t}$ and $x R_{c}^{z, t}(a) y$ and

- $f_{i+1}$ be the function that maps $W_{i+1}$ to $W^{\prime}$ such that, for all $x \in W_{i+1}$ :

- either $x \in W_{i}$ and $f_{i+1}(x)=f_{i}(x)$

- or there exists a defect $\left(c, z, t^{\prime}\right)$ of $\left(W_{i}, R_{i}\right)$ with respect to $\left(W^{\prime}, R^{\prime}\right)$ and $f_{i}$ such that $x \in W_{c}^{z, t}$ and $f_{i+1}(x)=h_{c}^{z, t}(x)$. 
The following facts hold :

Fact $1\left(W_{i+1}, R_{i+1}\right)$ is an irreflexive strong frame.

Proof : See the annex. $\dashv$

Fact $2 f_{i+1}$ is a surjective homomorphism of $\left(W_{i+1}, R_{i+1}\right)$ to $\left(W^{\prime}, R^{\prime}\right)$.

Proof : We leave the proof to the reader. $\dashv$

Fact 3 Let $\left(c, z, t^{\prime}\right)$ be a defect of $\left(W_{i}, R_{i}\right)$ with respect to $\left(W^{\prime}, R^{\prime}\right)$ and $f_{i}$. There exists $t \in W_{i+1}$ such that $z R_{i+1}(c) t$ and $f_{i+1}(t)=t^{\prime}$.

Proof : We leave the proof to the reader. $\dashv$ In conclusion, let :

- $W=\bigcup\left\{W_{i}: i \geq 0\right\}$,

- $R$ be the function that maps $\Pi_{c}$ to the set of the binary relations on $W$ such that, for all program $a$ and for all $x, y \in W$, there exists a positive integer $i$ such that $x, y \in W_{i}$ and $x R(a) y$ iff $x R_{i}(a) y$ and

- $f$ be the function that maps $W$ to $W^{\prime}$ such that, for all $x \in W$, there exists a positive integer $i$ such that $x \in W_{i}$ and $f(x)=f_{i}(x)$.

There is only one conclusion we can reach, which is that $(W, R)$ is an irreflexive strong frame and $f$ is a p-morphism of $(W, R)$ to $\left(W^{\prime}, R^{\prime}\right)$. $\dashv$

This only goes to show that:

Theorem 4 If a formula is valid in all strong models then it is valid in all weak models.

Proof : By lemmas 4 and 10. $\dashv$

Ultimately, then :

Corollary 1 Let $A$ be a formula. The following conditions are equivalent: $A$ is valid in all weak models, $A$ is a theorem of RMLCI, $A$ is a theorem of $R M L C I^{\prime}$ and $A$ is valid in all strong models.

Proof : By theorems 1, 2, 3 and 4. $\dashv$ 


\section{Conclusion}

In a previous paper "Complete axiomatization of a relative modal logic with composition and intersection", we have considered essentially the same syntactic characterization of the concept of validity in the standard Kripke semantics of $R M L C I$. Relying on the techniques of the subordination, we have demonstrated that the proof theory of $R M L C I$ is complete for the standard Kripke semantics of $R M L C I$. Our new proof of completeness for $R M L C I$ proceeds by showing that every weak frame is a p-morphic image of an irreflexive strong frame. With regard to this problem, given a weak frame $\left(W^{\prime}, R^{\prime}\right)$, we use mosaics as components to build up step by step an irreflexive strong frame $(W, R)$ and a p-morphism $f$ of $(W, R)$ to $\left(W^{\prime}, R^{\prime}\right)$. Undeniably, there is some difficulty in proving that mosaics are strong frames. However, the benefits of our new proof of completeness for $R M L C I$ will almost certainly outweigh the disadvantages. For the simple reason that it might be a good thing to consider frames such as mosaics to address the complexity issue of $R M L C I$ or to resolve the problem of the complete axiomatisation of IPDL. Questions that remain to be solved.

\section{Acknowledgement}

Special acknowledgement is heartly granted to Valentin Goranko, Andreas Herzig, Dimiter Vakarelov and the two anonymous referees who made several helpful comments for improving the readability of an earlier version of the paper.

\section{References}

[1] P. Balbiani, L. Fariñas del Cerro. Complete axiomatization of a relative modal logic with composition and intersection. Journal of Applied Non-Classical Logics, Volume 8, 325-335, 1998.

[2] D. Bredikhin, B. Schein. Representation of ordered semigroups and lattices by binary relations. Colloquium Mathematicum, Volume 39, 1$12,1978$. 
[3] R. Danecki. Nondeterministic propositional dynamic logic with intersection is decidable. A. Skowron (editor), Computation Theory. Lecture Notes in Computer Science 208, 34-53, Springer-Verlag, 1984.

[4] R. Fagin, J. Halpern, Y. Moses, M. Vardi. Reasoning About Knowledge. MIT Press, 1995.

[5] L. Fariñas del Cerro, E. Orłowska. DAL - a logic for data analysis. Theoretical Computer Science, Volume 36, 251-264, 1985.

[6] M. Fischer, R. Ladner. Propositional dynamic logic of regular programs. Journal of Computer and System Sciences, Volume 18, 194-211, 1979.

[7] G. Gargov, S. Passy. A note on Boolean modal logic. P. Petkov (editor), Mathematical Logic. 299-309, Plenum Press, 1990.

[8] D. Harel. Recurring dominoes : making the highly undecidable highly understandable. M. Karpinski (editor), Foundations of Computation Theory. Lecture Notes in Computer Science 158, 177-194, SpringerVerlag, 1983.

[9] G. Hughes, M. Cresswell. A Companion to Modal Logic. Methuen, 1984.

[10] M. Marx, Y. Venema. Multi-Dimensional Modal Logic. Kluwer, 1997.

[11] J.-J. Meyer. A different approach to deontic logic : deontic logic viewed as a variant of dynamic logic. Notre Dame Journal of Formal Logic, Volume 29, 109-136, 1988.

[12] G. Mirkowska. PAL - propositional algorithmic logic. Fundamenta Informaticæ, Volume 4, 675-760, 1981.

[13] E. Orłowska. Kripke models with relative accessibility and their applications to inferences from incomplete information. Banach Center Publications, Mathematical Problems in Computation Theory. Volume 21, 329-339, Polish Scientific Publishers, 1988.

[14] S. Passy, T. Tinchev. An essay in combinatory dynamic logic. Information and Control, Volume 93, 263-332, 1991. 


\section{Annex}

Proof of the fact 1 : The easy proof that $\left(W_{i+1}, R_{i+1}\right)$ is an irreflexive frame such that $R_{i+1}$ satisfies the conditions : $R_{i+1}(a ; b) \supseteq R_{i+1}(a) \circ R_{i+1}(b)$ and $R_{i+1}(a \wedge b)=R_{i+1}(a) \cap R_{i+1}(b)$ is left to the reader. Let us demonstrate that, for all program $a$, for all real numbers $u, v, u \neq v$, and for all $x, y \in$ $W_{i+1}, x R_{i+1}(a) y$, there exists a homomorphism $h$ of $\iota(a, u, v)$ to $\left(W_{i+1}, R_{i+1}\right)$ such that $h(u)=x$ and $h(v)=y$. We may assume that, for all program $a$, for all real numbers $u, v, u \neq v$, and for all $x, y \in W_{i}, x R_{i}(a) y$, there exists a homomorphism $h$ of $\downarrow(a, u, v)$ to $\left(W_{i}, R_{i}\right)$ such that $h(u)=x$ and $h(v)=y$. Let $a$ be a program, $u, v$ be real numbers, $u \neq v$, and $x, y \in W_{i+1}, x R_{i+1}(a)$ $y$. Then we have to consider three cases.

Case $1: x, y \in W_{i}$ and $x R_{i}(a) y$. According to our opening hypothesis, there exists a homomorphism $h$ of $\natural(a, u, v)$ to $\left(W_{i}, R_{i}\right)$ such that $h(u)=x$ and $h(v)=y$. The reader may easily verify that $h$ is a homomorphism of $\natural(a, u, v)$ to $\left(W_{i+1}, R_{i+1}\right)$.

Case 2 : There exists a defect $\left(c, z, t^{\prime}\right)$ of $\left(W_{i}, R_{i}\right)$ with respect to $\left(W^{\prime}, R^{\prime}\right)$ and $f_{i}$ such that $x \in W_{i} \backslash\{z\}, y \in W_{c}^{z, t} \backslash\{z\}$ and there exist programs $a^{\prime}, a^{\prime \prime}$ such that $x R_{i}\left(a^{\prime}\right) z, z R_{c}^{z, t}\left(a^{\prime \prime}\right) y$ and $a^{\prime} ; a^{\prime \prime} \leq a$. Let $\downarrow\left(a^{\prime} ; a^{\prime \prime}, u, v\right)$ be the frame of the form $\left(W_{a^{\prime} ; a^{\prime \prime}}, R_{a^{\prime} ; a^{\prime \prime}}\right)$ where $w$ is a new real number, $\downarrow\left(a^{\prime}, u, w\right)=$ $\left(W_{a^{\prime}}, R_{a^{\prime}}\right), \downarrow\left(a^{\prime \prime}, w, v\right)=\left(W_{a^{\prime \prime}}, R_{a^{\prime \prime}}\right), W_{a^{\prime} ; a^{\prime \prime}}=W_{a^{\prime}} \cup W_{a^{\prime \prime}}$ and, for all program $d$ and for all $p, q \in W_{a^{\prime} ; a^{\prime \prime}}, p R_{a^{\prime} ; a^{\prime \prime}}(d) q$ iff :

- either $p, q \in W_{a^{\prime}}$ and $p R_{a^{\prime}}(d) q$

- or $p \in W_{a^{\prime}} \backslash\{w\}, q \in W_{a^{\prime \prime}} \backslash\{w\}$ and there exist programs $d^{\prime}, d^{\prime \prime}$ such that $p R_{a^{\prime}}\left(d^{\prime}\right) w, w R_{a^{\prime \prime}}\left(d^{\prime \prime}\right) q$ and $d^{\prime} ; d^{\prime \prime} \leq d$

- or $p, q \in W_{a^{\prime \prime}}$ and $p R_{a^{\prime \prime}}(d) q$.

According to our opening hypothesis, there exists a homomorphism $h_{a^{\prime}}$ of $\left(W_{a^{\prime}}, R_{a^{\prime}}\right)$ to $\left(W_{i}, R_{i}\right)$ such that $h_{a^{\prime}}(u)=x$ and $h_{a^{\prime}}(w)=z$. By lemma 8 , there exists a homomorphism $h_{a^{\prime \prime}}$ of $\left(W_{a^{\prime \prime}}, R_{a^{\prime \prime}}\right)$ to $\left(W_{c}^{z, t}, R_{c}^{z, t}\right)$ such that $h_{a^{\prime \prime}}(w)=$ $z$ and $h_{a^{\prime \prime}}(v)=y$. Let $h_{a^{\prime} ; a^{\prime \prime}}$ be the mapping of $W_{a^{\prime} ; a^{\prime \prime}}$ to $W_{i+1}$ such that $h_{a^{\prime} ; a^{\prime \prime} \mid W_{a^{\prime}}}=h_{a^{\prime}}$ and $h_{a^{\prime} ; a^{\prime \prime} \mid W_{a^{\prime \prime}}}=h_{a^{\prime \prime}}$. The reader may easily verify that $h_{a^{\prime} ; a^{\prime \prime}}$ is a homomorphism of $\left(W_{a^{\prime} ; a^{\prime \prime}}, R_{a^{\prime} ; a^{\prime \prime}}\right)$ to $\left(W_{i+1}, R_{i+1}\right)$ such that $h_{a^{\prime} ; a^{\prime \prime}}(u)=x$ and $h_{a^{\prime} ; a^{\prime \prime}}(v)=y$. By lemma 7 , there exists a homomorphism $h$ of $\downarrow(a, u, v)$ to $\left(W_{a^{\prime} ; a^{\prime \prime}}, R_{a^{\prime} ; a^{\prime \prime}}\right)$ such that $h(u)=u$ and $h(v)=v$. Then $h \circ h_{a^{\prime} ; a^{\prime \prime}}$ is a 
homomorphism of $\natural(a, u, v)$ to $\left(W_{i+1}, R_{i+1}\right)$ such tha $\left(h \circ h_{a^{\prime} ; a^{\prime \prime}}\right)(u)=x$ and $\left(h \circ h_{a^{\prime} ; a^{\prime \prime}}\right)(v)=y$.

Case 3 : There exists a defect $\left(c, z, t^{\prime}\right)$ of $\left(W_{i}, R_{i}\right)$ with respect to $\left(W^{\prime}, R^{\prime}\right)$ and $f_{i}$ such that $x, y \in W_{c}^{z, t}$ and $x R_{c}^{z, t}(a) y$. By lemma 8, there exists a homomorphism $h$ of $\downarrow(a, u, v)$ to $\left(W_{c}^{z, t}, R_{c}^{z, t}\right)$ such that $h(u)=x$ and $h(v)=$ $y$. The reader may easily verify that $h$ is a homomorphism of $\downarrow(a, u, v)$ to $\left(W_{i+1}, R_{i+1}\right)$.

Let us demonstrate that $R_{i+1}$ satisfies the condition : $R_{i+1}(a ; b) \subseteq R_{i+1}(a) \circ$ $R_{i+1}(b)$. Let $a, b$ be programs and $x, y \in W_{i+1}, x R_{i+1}(a ; b) y$. Suppose now that, for all $z \in W_{i+1}, x \overline{R_{i+1}(a)} z$ or $z \overline{R_{i+1}(b)} y$. Let $u, v$ be real numbers, $u \neq v$. Let $\downarrow(a ; b, u, v)$ be the frame of the form $\left(W_{a ; b}, R_{a ; b}\right)$ where $w$ is a new real number, $\natural(a, u, w)=\left(W_{a}, R_{a}\right), \natural(b, w, v)=\left(W_{b}, R_{b}\right), W_{a ; b}=W_{a} \cup W_{b}$ and, for all program $d$ and for all $p, q \in W_{a ; b}, p R_{a ; b}(d) q$ iff :

- either $p, q \in W_{a}$ and $p R_{a}(d) q$

- or $p \in W_{a} \backslash\{w\}, q \in W_{b} \backslash\{w\}$ and there exist programs $d^{\prime}, d^{\prime \prime}$ such that $p R_{a}\left(d^{\prime}\right) w, w R_{b}\left(d^{\prime \prime}\right) q$ and $d^{\prime} ; d^{\prime \prime} \leq d$

- or $p, q \in W_{b}$ and $p R_{b}(d) q$.

Then there exists a homomorphism $h$ of $\left(W_{a ; b}, R_{a ; b}\right)$ to $\left(W_{i+1}, R_{i+1}\right)$ such that $h(u)=x$ and $h(v)=y$. Since $u R_{a}(a) w$ then $u R_{a ; b}(a) w$. Since $w R_{b}(b) v$ then $w R_{a ; b}(b) v$. Then $x R_{i+1}(a) h(w)$ and $h(w) R_{i+1}(b) y$, a contradiction. $\dashv$ 